\title{
Diffusion multiple study of the Co-Fe-Ni system at $800{ }^{\circ} \mathrm{C}$
}

\author{
Yuan Yuan ${ }^{1,2,3 *}$, Tao Chen ${ }^{1}$, Dajian $\mathrm{Li}^{3}$, Uta Gerhards ${ }^{4}$, Fusheng Pan ${ }^{1}$, Hans Seifert ${ }^{3}$, \\ Nele Moelans ${ }^{2}$ \\ ${ }^{1}$ College of Materials Science and Engineering, Chongqing University, Chongqing 400000, China \\ ${ }^{2}$ KU Leuven, Department of Materials Engineering, Leuven, 3001, Belgium \\ ${ }^{3}$ Karlsruhe Institute of Technology, Institute for Applied Materials - Applied Materials Physics (IAM- \\ AWP), Karlsruhe, 76344, Germany \\ ${ }^{4}$ Karlsruhe Institute of Technology, Institute for Micro Process Engineering (IMVT), Karlsruhe, \\ 76344, Germany
}

Postprint of article published as "CALPHAD-COMPUTER COUPLING OF PHASE DIAGRAMS AND THERMOCHEMISTRY 64:149-159 01, Mar 2019”

\begin{abstract}
The Co-Fe-Ni system is a key system in a wide range of industrial applications. Knowledge of the thermodynamic and kinetic properties of the system is crucial for the alloy and process design. Although the system has been studied extensively, there remain several unexplained discrepancies between different literature data and, for mediate low temperatures, the information is scarce. In this work, a high throughput diffusion multiple approach was applied. The isothermal phase diagram section at $800{ }^{\circ} \mathrm{C}$ was determined using the Co-Fe-Ni multiple. The interdiffusion coefficients of the binary $\mathrm{Co}-\mathrm{Fe}, \mathrm{Co}-\mathrm{Ni}, \mathrm{Fe}-\mathrm{Ni}$ systems and their composition dependence were calculated using the Sauer-Freise method based on the compositional profiles obtained from the diffusion multiple. Different from previous experimental results for mediate low temperature, our results coincide with the extrapolated Arrhenius temperature dependence from diffusion coefficient data at high temperature range. These observations are important for a better understanding and modelling of the interdiffusion behaviour in this key alloy system.
\end{abstract}

Key words: Co-Fe-Ni system; isothermal section; diffusion multiple; interdiffusion coefficients 


\section{Introduction}

Co-Fe-Ni, as well as, its sub-binary systems are essential for many industrial fields, such as steel [1], high entropy alloy [2] and high temperature alloy. Correspondingly, phase diagrams, thermodynamic and kinetic information are crucial for the alloy and processing design. No ternary intermediate phase exists in this system. The three elements are mutual soluble in a large composition range. Fig. 1 shows the phase diagrams of the three sub-binary systems. The ternary Co-Fe-Ni system was assessed by Guillermet [3], where the two phase fcc+bcc region is an important feature in this ternary system. Raghavan [4] point out the discrepancy of the boundary of the region of fcc+bcc between experimental results [5,6] and assessed results [3] and the necessities of further experimental work. Recently, Xing et al [7] determined isothermal sections at $500{ }^{\circ} \mathrm{C}, 600{ }^{\circ} \mathrm{C}, 700{ }^{\circ} \mathrm{C}$ using the combinatorial materials chip approach, where the discrepancies with previously assessed data [3] were also present.

Many groups have studied the diffusion in Co-Fe-Ni system and its sub-systems [8-23], in which most studies focused on the fcc phase in the high temperature range. Heumann and Kottmann [17] studied interdiffusion of the fcc phase in the Fe-Ni system at $1310^{\circ} \mathrm{C}$ and in the Co-Ni system at $1155^{\circ} \mathrm{C}, 1305^{\circ} \mathrm{C}$ and $1400{ }^{\circ} \mathrm{C}$ using diffusion couple technique and calculated the corresponding interdiffusion coefficients using Bolztmann-Matano method. Similarly, Ustad and Sorum [14] determined the interdiffusion coefficients and corresponding activation energies of the $\mathrm{Fe}-\mathrm{Ni}$, and $\mathrm{Co}-$ Fe systems in the temperature range of $705-1400{ }^{\circ} \mathrm{C}$ and of the $\mathrm{Co}-\mathrm{Ni}$ system in the temperature range of $1000-1400{ }^{\circ} \mathrm{C}$. In their results, consistent data in the temperature range of 1000 to $1400{ }^{\circ} \mathrm{C}$ were observed. There was, however, a discrepancy for the data at mediate low temperatures of $705^{\circ} \mathrm{C}$ and $805^{\circ} \mathrm{C}$, as these data did not follow the Arrhenius relation. They attribute the discrepancy to a change in diffusion mechanism with temperature, which is, at high temperatures, lattice diffusion and, at lower temperatures, grain boundary diffusion. Ugaste et al studied the interdiffusion and Kirkendall effect in the binary Co-Ni, Fe-Ni and Co-Fe systems at $1000-1300{ }^{\circ} \mathrm{C}[8,12]$, and in the ternary Co-Fe-Ni system $[11,13,24]$ covering a large composition range. 
Based on all these experimental results, Cui et al [9,25] assessed the experimental data and obtained the corresponding kinetic parameters based on the kinetic model developed by Agren [26]. However, the assessment doesn't go to the low temperature range because of the discrepancy in the experimental data $[14,18]$.

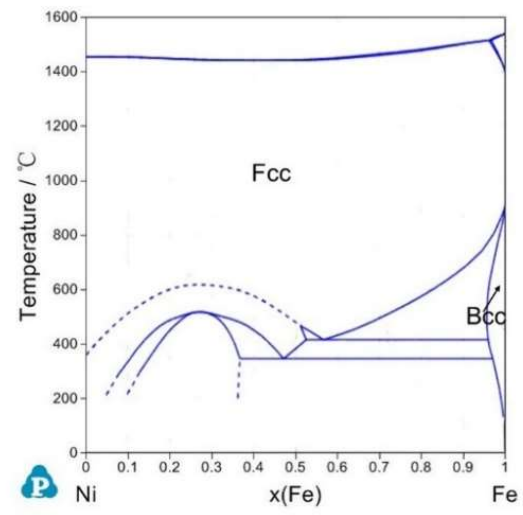

(a) Ni-Fe system

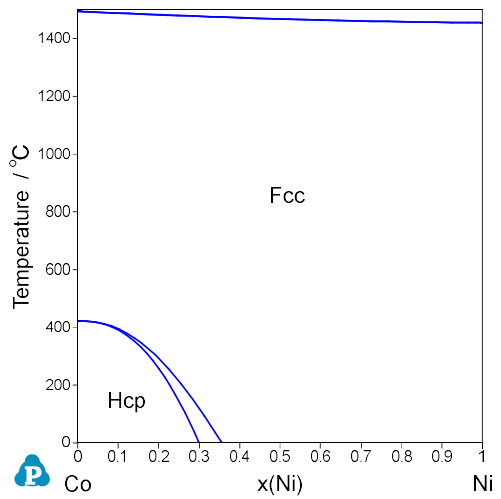

(b) Co-Ni System

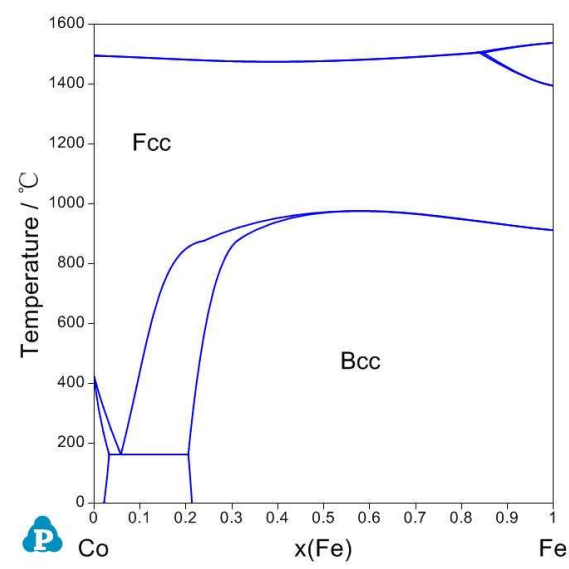

(c) Co-Fe system

Fig. 1 Phase diagrams of the corresponding systems calculated using PanFe database: (a) FeNi system, (b) Co-Ni system, (c) Co-Fe system.

Therefore, the aim of the current work is to investigate the isothermal section of the Co-Fe-Ni phase diagram and the interdiffusion in the three binary systems at $800{ }^{\circ} \mathrm{C}$ using diffusion multiple approach. A diffusion multiple of Co-Fe-Ni-Ti were constructed in this work. The work results of Ti-Ni-Co system on this diffusion multiple were published as another work [27]. 


\section{Experimental Processes}

High-throughput diffusion multiple approach [28], which have been widely applied for studying interdiffusion behavior [29-32], was applied in the current work. Plates with dimension of $5 \mathrm{~mm} \times 1 \mathrm{~mm} \times 10 \mathrm{~mm}$ were cut from high purity $\mathrm{Fe}, \mathrm{Co}, \mathrm{Ti}$ and Ag (99.999 mass\%, NewMet) ingots. After polishing and cleaning with ethanol in an ultrasonic bath to obtain flat and clean surfaces, the plates were inserted into a high purity $\mathrm{Ni}$ ( 99.999 mass\%, NewMet) cartridge to avoid the contamination from any other materials. Hence, a diffusion multiple including Ni, Ti, Fe and $\mathrm{Co}$ is assembled. This diffusion multiple was then inserted into a can made from commercial $\mathrm{Ni}$ and lastly, inserted in a $\mathrm{Ti}$ can specified for the HIP (high temperature iso-static pressing) processing. Then the Ti can was sealed using electron beam welding. The complete setup is shown in Fig. 2, where a scale to indicate the dimension of the whole assembly were provided.

High temperature iso-static pressing treatment of the whole assembly was performed at $800^{\circ} \mathrm{C}$ for 4 hours with pressure $20 \mathrm{MPa}$. The process is illustrated in Fig. 3. Afterwards, the whole assembly was sealed in quartz ampoule, annealed at $800{ }^{\circ} \mathrm{C}$ for 284 hours (in total 288 hours of diffusion including the HIP time 4 hours) in a muffle furnace and water quenched. Finally, the assembly was cut perpendicular in the middle, grinded, polished, and ultrasonically cleaned using standard metallographic techniques.

Philips XL30 FEG scanning electron microscope was used to analyze the phase structure and morphology of the diffusion zone. The morphology of the diffusion zone was analyzed using Back Scatter Electron (BSE) mode. Phase composition of the diffusion layer was analyzed using field emission electron probe micro-analyzer (EPMA) under wavelength dispersive spectroscopy (WDS) mode. Quantitative linescanning module with step size of 0.5 or $1 \mu \mathrm{m}$ was utilized to obtain the concentration profiles in the $\mathrm{Fe}-\mathrm{Ni}, \mathrm{Fe}-\mathrm{Co}$, Ni-Co diffusion layers. For each binary diffusion layer, three or four lines perpendicular to the interface were selected for the quantitative line scanning. 


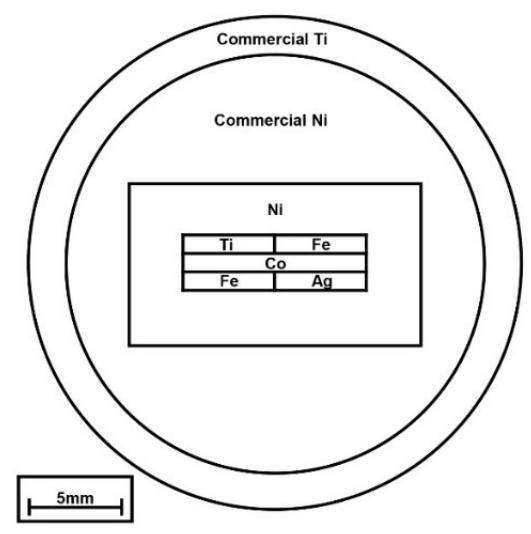

Fig. 2 The scheme of the multicomponent diffusion assembly.

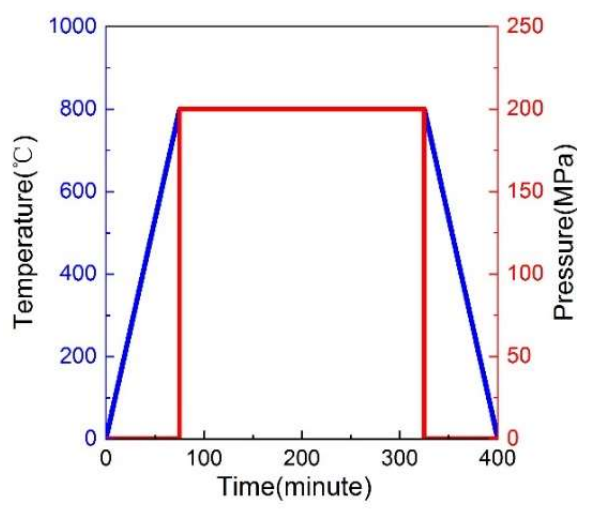

Fig. 3 The high temperature iso-static pressing treatment of the diffusion multiple assembly

\section{Diffusion calculation method}

The interdiffusion coefficient describing the intermixing effect due to diffusion, $\widetilde{D}$, can be determined from the composition profile measured in the diffusion couples [33]. The most widely applied method to determine the interdiffusion coefficient is the Boltzmann-Matano method [34]. Sauer and Freise [35] imported an auxiliary variable $Y(x)$ into the Boltzmann-Matano method to avoid the need of the evaluation of the position of the Matano plane. $Y(x)$ is defined by equation (1):

$$
Y(x)=\frac{N_{i}(x)-N_{i}^{+\infty}}{N_{i}^{+\infty}-N_{i}^{-\infty}}
$$

where $N_{i}(x)$ is the mole fraction of specie $i$ at the position $x$; superscripts $+\infty$ and $-\infty$ denote the corresponding values in the left and right end-boundaries of the diffusion couple, respectively.

The interdiffusion coefficient $\widetilde{D}$ at a certain point $x^{*}$ in the diffusion zone can then be evaluated from 


$$
\widetilde{D}\left(Y^{*}\right)=\frac{V_{m}}{2 t\left(\frac{d Y}{d x}\right)_{x^{*}}}\left[\left(1-Y^{*}\right) \int_{x^{-\infty}}^{x^{*}} \frac{Y}{V_{m}} d x+Y^{*} \int_{x^{*}}^{x^{+\infty}} \frac{1-Y}{V_{m}} d x\right]
$$

where the superscript * denotes the corresponding value at the investigated point $x^{*}$; and $V_{m}$ is the volume of one mole atoms, which is assumed be same for the investigated system in this work.

The temperature dependence of interdiffusion coefficients is expected to follow a Arrhenius equation (3) over extended temperature ranges [33]:

$$
\widetilde{D}=D_{0} \exp (-\mathrm{Q} / \mathrm{RT})
$$

where $\mathrm{Q}$ is activation energy; $D_{0}$ is a frequency factor; $\mathrm{R}$ is gas constant; and $\boldsymbol{T}$ is absolute temperature. Using equation (3), the obtained interdiffusion coefficients can be extrapolated to other temperatures.

\section{Results and discussion}

\subsection{Diffusion coefficients of the Fe-Ni system}

Fig. 4 shows a BSE image of the binary Fe-Ni joint (a), the Composition profile of the Fe-Ni diffusion layer (b) and the calculated interdiffusion coefficients at $800{ }^{\circ} \mathrm{C}$ (c). Note that the obtained interdiffusion coefficients for compositions near the pure elements are with large uncertainty. This is because of the relative slow change and hence scattered data of composition at the boundaries can result in a large error when calculating the composition gradient. 


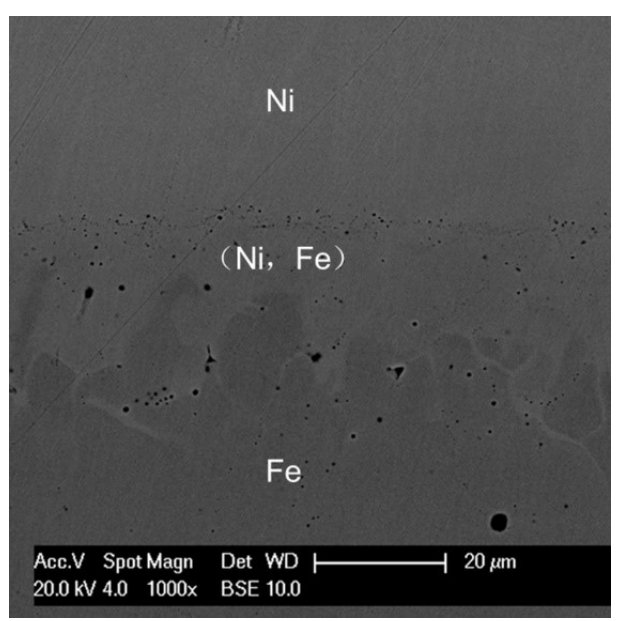

(a)

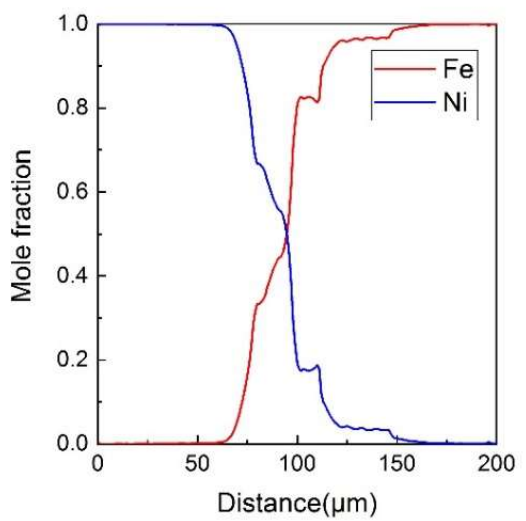

(b)

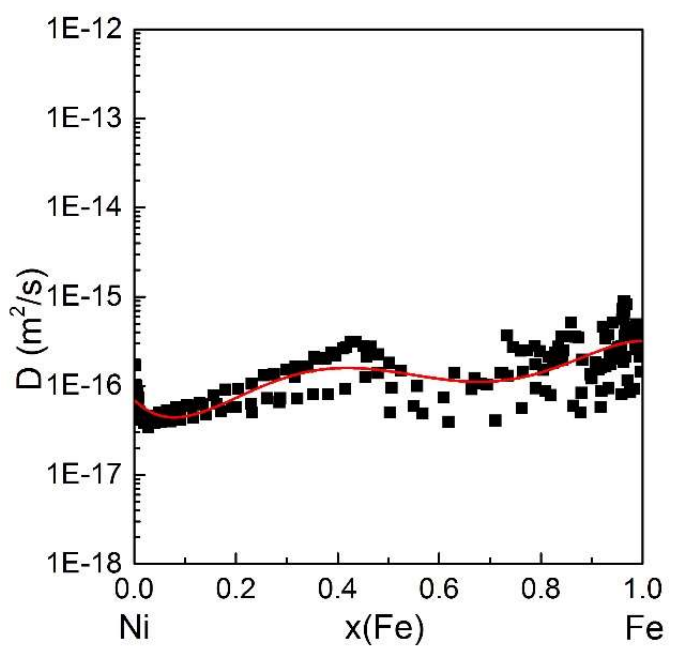

(c)

Fig. 4 (a) BSE image of the Fe-Ni binary joint at $800{ }^{\circ} \mathrm{C}$;

(b) Measured composition profile of the Fe-Ni diffusion layer of one typical line perpendicular to the interface;

(c) interdiffusion coefficients calculated based on the measured composition profile (b) at $800{ }^{\circ} \mathrm{C}$. In (c) black points represent the data calculated from several lines perpendicular to the interface and the red line is the fitting curve using the least squares fitting method.

The interdiffusion coefficients at different compositions are compared to literature data [14,15] in Fig. 5. Interestingly, the experimental results from Ustad et al. [14] follow an Arrhenius equation in the high-temperature region (above $1300{ }^{\circ} \mathrm{C}$ ), but show a deviation from this relation for the lower temperature range. However, our $\widetilde{D}$ results show a general agreement to the Arrhenius line determined by the high 
temperature results from Ustad et al. [14]. According to Fig. 1(a), the phase region is changed to $\alpha+\gamma$ two phase region as $\mathrm{Fe}-\mathrm{Ni}$ alloys at around 90 at $\% \mathrm{Fe}$ at $800{ }^{\circ} \mathrm{C}$, which could contribute to the larger spread on the experimental data in this region. Here the multi-grain material blocks were employed for the studies. The calculated interdiffusion data at $800^{\circ} \mathrm{C}$ shown perfect consistent with the extrapolated data from high temperature range, hence the lattice diffusion mechanism is still believed to be the main diffusion type at this temperature

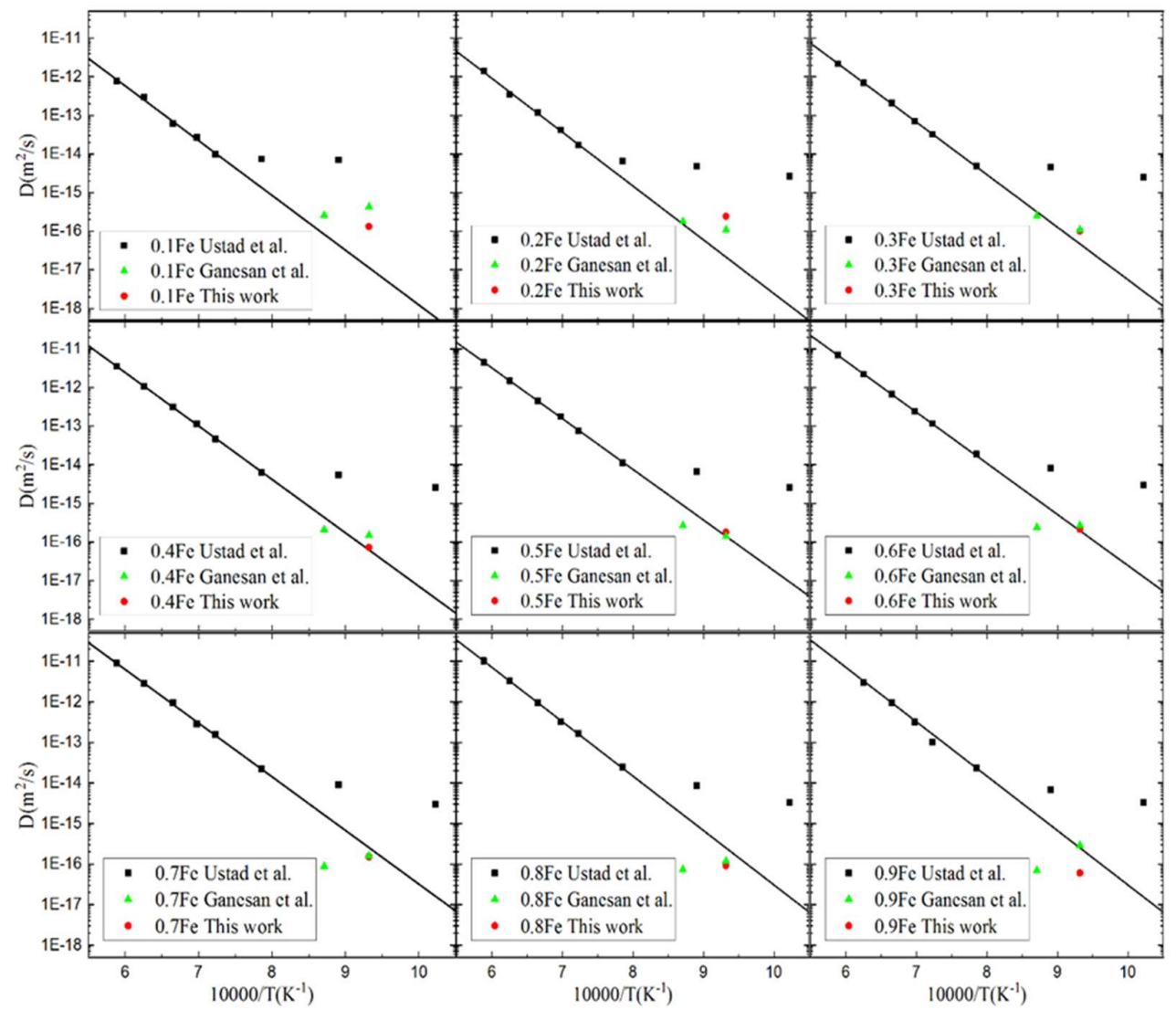

Fig. 5 Interdiffusion coefficients versus reciprocal temperature of the Fe-Ni system and compared with the data from Ustad et al [14] and Ganesan et al [15].

\subsection{Diffusion of the Co-Ni system}




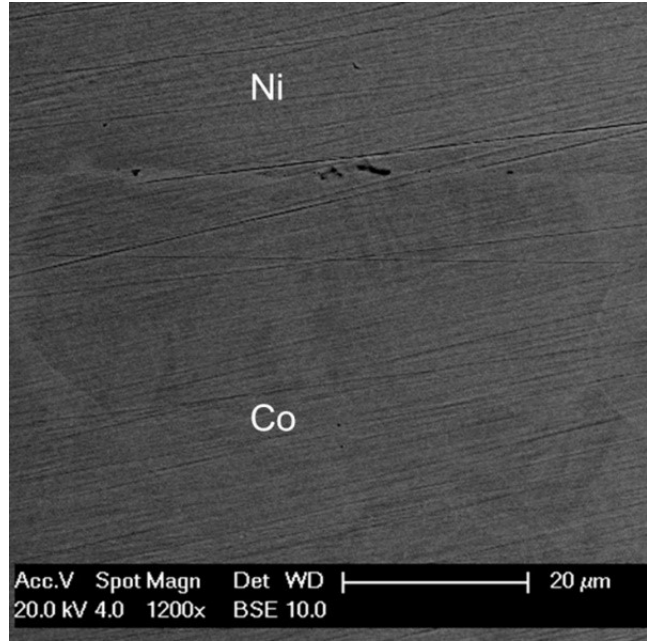

(a)

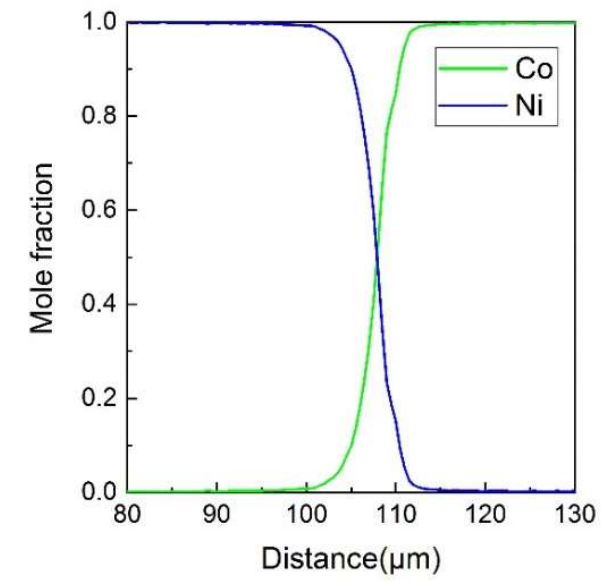

(b)

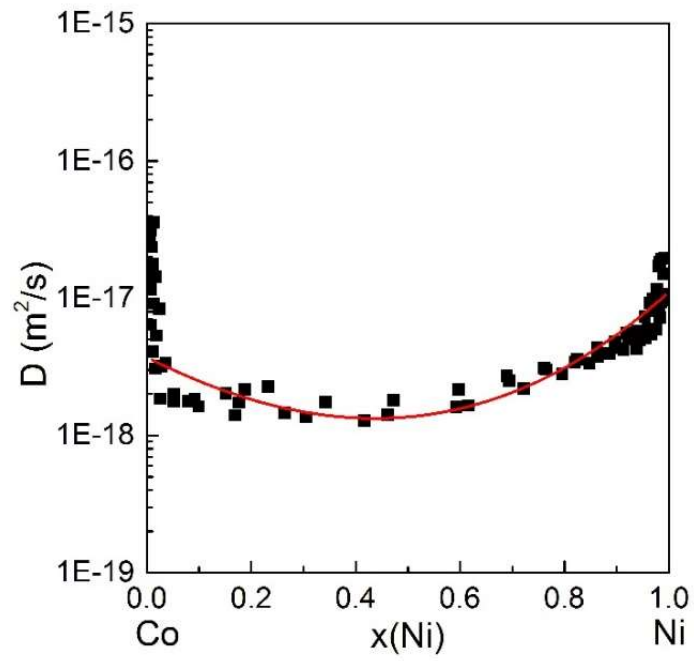

(c)

Fig. 6 (a) BSE image of the Co-Ni binary joint at $800{ }^{\circ} \mathrm{C}$;

(b) Measured composition profile of the Co-Ni diffusion layer of one typical line perpendicular to the interface;

(c) Interdiffusion coefficients calculated based on the measured composition profile (b) at $800{ }^{\circ} \mathrm{C}$. In (c) black points represent the data calculated from several lines perpendicular to the interface and the red line is the fitting curve using the least squares fitting method.

Fig. 6 shows a BSE image of the Co-Ni binary joint (a), the measured composition profile in the Co-Ni diffusion zone (b), and calculated diffusion coefficients for the Co$\mathrm{Ni}$ system at $800^{\circ} \mathrm{C}(\mathrm{c})$, respectively. 
Heumann ang Kottmano [17], Iijina and Hirano [36], Kucera et al. [20] and Ustad and Sorum [14] measured interdiffusion coefficients of the Co-Ni system. Similarly to the Fe-Ni system, the results from Ustad and Sorum [14] show a deviation from the Arrhenius relation extrapolated from higher temperature data for temperatures below $1300{ }^{\circ} \mathrm{C}$. However, our values are perfectly in line with the Arrhenius relation extrapolated from higher temperature data in literature, as shown in Fig. 7. Furthermore, the change of the interdiffusion coefficients with the composition dependence is found to be quite small. This is physically reasonable since the two elements are completely mutual soluble, as shown in Fig. 1 (b), and the atomic radiuses of two elements are similar.

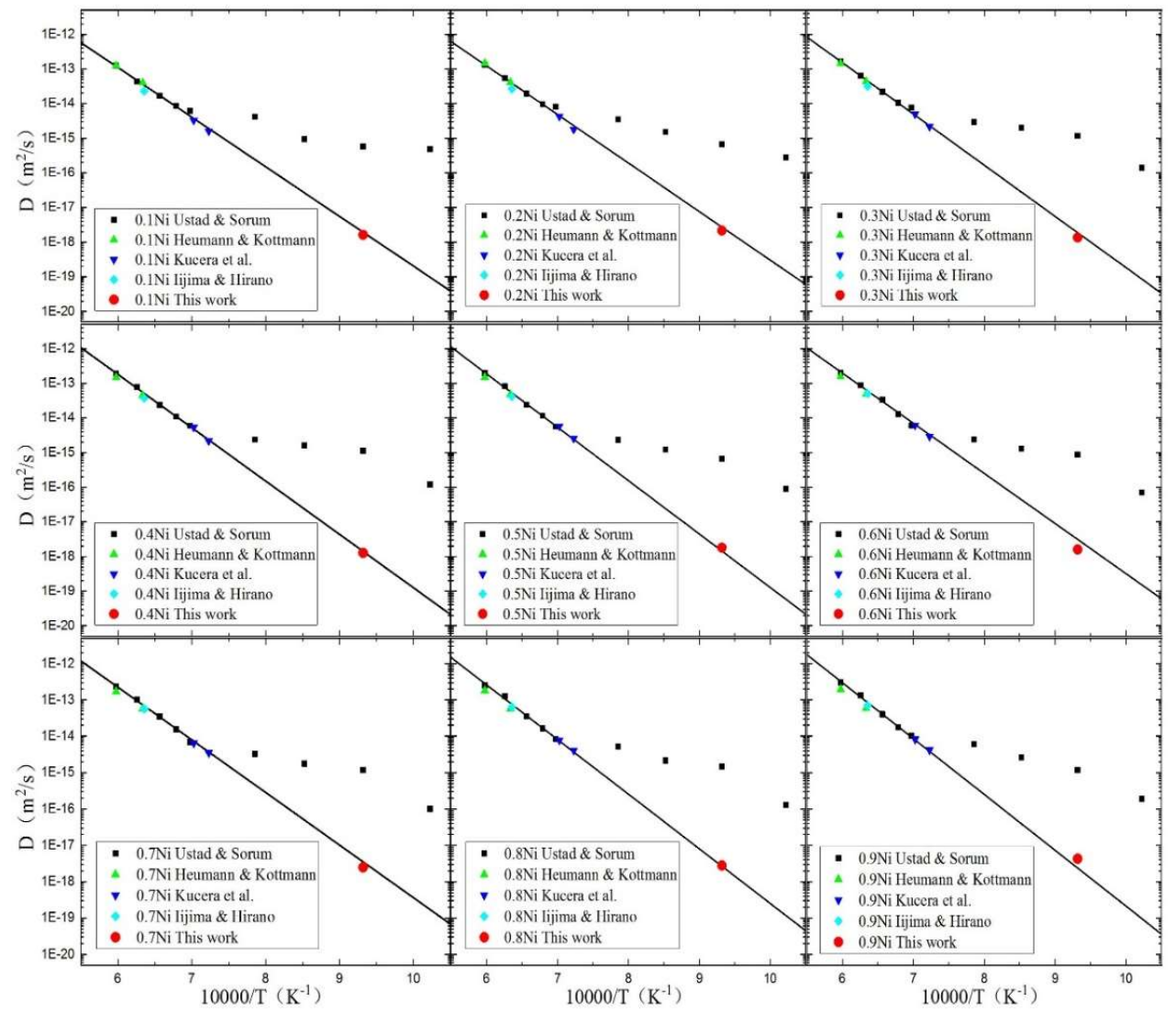

Fig. 7: The Interdiffusion coefficient versus reciprocal temperature of the Co-Ni system and compared with literature data from Heumann ang Kottmano [15], Iijina and Hirano [34], Kucera et al. [18] and Ustad and Sorum [12]. 


\subsection{Diffusion of the Co-Fe system}
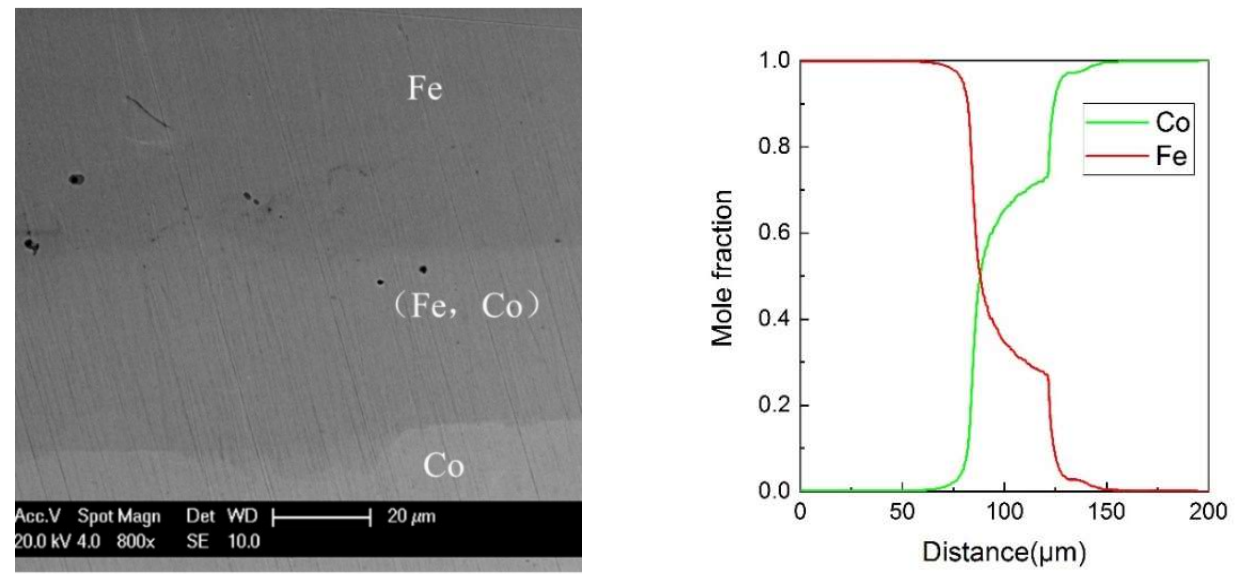

(a)

(b)

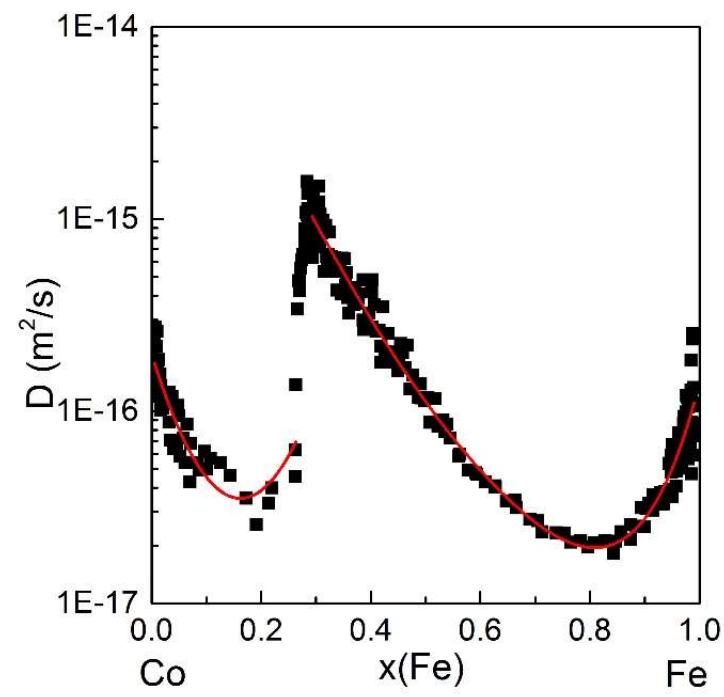

(c)

Fig. 8 (a) BSE image of the Co-Fe binary joint at $800{ }^{\circ} \mathrm{C}$;

(b) Measured composition profile of the Co-Fe diffusion layer of one typical line perpendicular to the interface;

(c) Interdiffusion coefficients calculated based on the measured composition profile (b) at $800{ }^{\circ} \mathrm{C}$. In (c) black points represent the data calculated from several lines perpendicular to the interface and the red line is the fitting curve using the least squares fitting method. 
Fig. 8 shows BSE image of Fe-Co binary joint (a), the composition profile in the $\mathrm{Fe}-\mathrm{Co}$ diffusion zone (b) and calculated diffusion coefficient of Fe-Co system at $800^{\circ} \mathrm{C}$ (c).

At $800{ }^{\circ} \mathrm{C}$, both bcc and fcc phases presented with the change of composition. Accordingly, the diffusion curve can be divided into two parts. Ustad et al. [14] and Badia et al. [37] investigated the diffusion in fcc Fe-Co above $1000{ }^{\circ} \mathrm{C}$, which is above the temperatures of the fcc-bcc phase transition. Fig. 9 shows the interdiffusion coefficients versus reciprocal temperature of the Fe-Co system. It is seen that the values of $\widetilde{D}$ at temperature of $800{ }^{\circ} \mathrm{C}$ obtained in this work coincide with the Arrhenius line extrapolated from the literature data [14,37] for both fcc and bec phase, excluding the points at $30 \mathrm{at} \% \mathrm{Fe}$ and $40 \mathrm{at} \% \mathrm{Fe}$. These two composition points, as shown in Fig. 1(c), are in or near the two-phase region and the values obtained at this region was considered to be less reliable. The sharp change of the composition at the phase interface, as shown in Fig. 8(b), may results a sharp change of the gradient, as well as, a sharp change of the diffusion constants. A strong composition dependence of the interdiffusion curve for both phases was observed. 


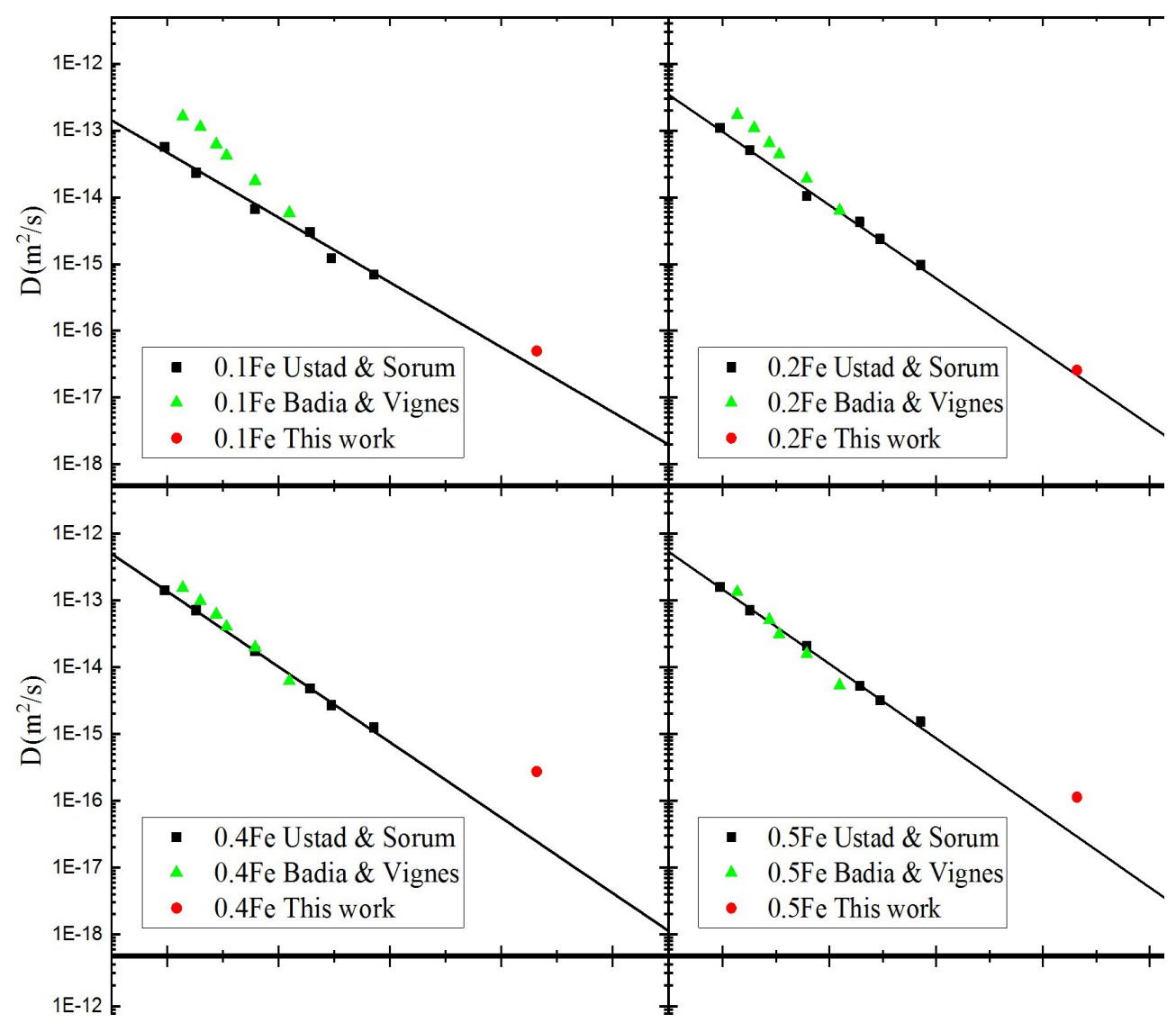

Fig. 9 Interdiffusion coefficients versus reciprocal temperature of the Co-Fe system and compared with the data from Ustad et al.[14] and Badia et al. [37].

\subsection{The isothermal section of the Fe-Co-Ni system at $800{ }^{\circ} \mathrm{C}$}

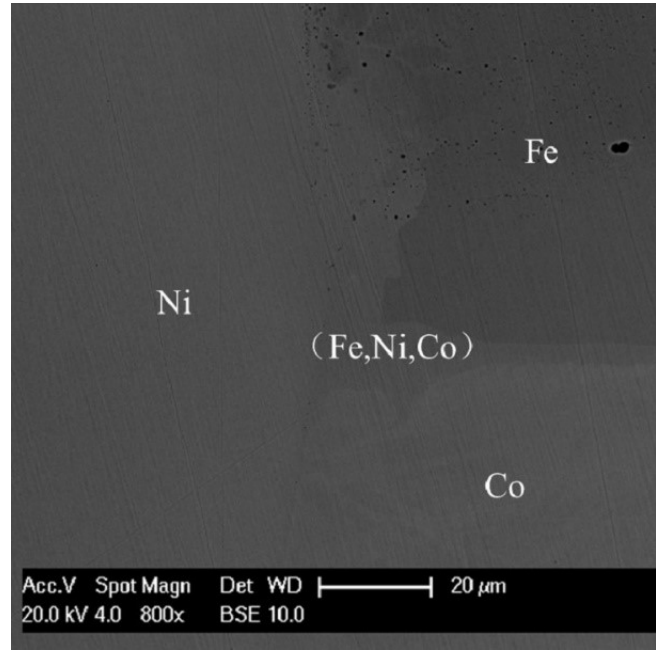

(a)

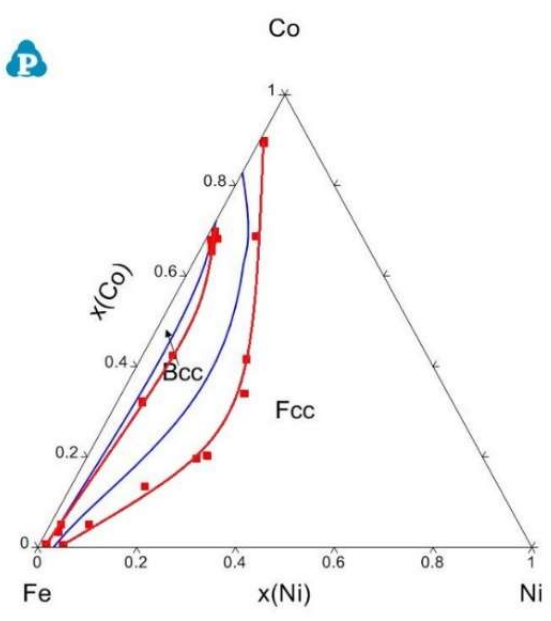

(b)

Fig. 10 (a) The obtained BSE image of the Co-Fe-Ni ternary joint at $800{ }^{\circ} \mathrm{C}$

(b) The isothermal section of the $\mathrm{Co}-\mathrm{Fe}-\mathrm{Ni}$ system at $800^{\circ} \mathrm{C}$ 
Microscopy image of the ternary joint and the schemed isothermal-section is given in Fig. 10. No intermediate phases show up in the Co-Fe-Ni system. Mutual diffusion among the three elements has been observed in the diffusion region. According to the EPMA analysis, the schemed phase regions at the section of $800{ }^{\circ} \mathrm{C}$ is shown in Fig. 10 (b), in which the blue lines are the phase boundaries calculated using assessment database [3]. For the boundary of the two-phase fcc+bcc region, a clear discrepancy between the calculation and this experimental work can be observed. Re-assessment of the system is therefore suggested since this system is so important and broadly used.

\section{Conclusion}

Co-Fe-Ni system is a key system in many fields of industrial applications. The phase equilibria and binary diffusion coefficients of the $\mathrm{Co}-\mathrm{Fe}-\mathrm{Ni}$ system at $800^{\circ} \mathrm{C}$ were investigated using the diffusion multiple approach.

The isothermal section at $800^{\circ} \mathrm{C}$ was obtained based on a ternary diffusion joint, where the two-phase region of fcctbcc is determined and a discrepancy with the assessed data is observed.

Reliable interdiffusion coefficient of Co-Fe, Fe-Ni and Co-Ni systems at $800^{\circ} \mathrm{C}$ is obtained. The interdiffusion coefficients are perfectly in coincident with the literature data in high temperature range extrapolated to the low temperature following the Arrhenius equation.

\section{Acknowledgement}

Authors thank the group-starting fund from the Fundamental Research Funds for the Chinese Central Universities of Ministry of Education of China. The corresponding author, Yuan Yuan, is a Pegasus Marie Curie Fellow of the Research Foundation Flanders (FWO - Vlaanderen), Belgium, and European Commission, Europe. The funding is in the European Union's Seventh Framework Program for research, technological development and demonstration. 


\section{Data availability}

The datasets generated during and/or analyzed during the current study are available on request.

\section{Reference}

[1] J. Tian, M.B. Shahzad, W. Wang, L. Yin, Z. Jiang, K. Yang, Role of Co in formation of Ni-Ti clusters in maraging stainless steel, J. Mater. Sci. Technol. 34 (2018) 1671-1675. doi:10.1016/J.JMST.2018.04.020.

[2] R. Wang, W. Chen, J. Zhong, L. Zhang, Experimental and numerical studies on the sluggish diffusion in face centered cubic Co-Cr-Cu-Fe-Ni high-entropy alloys, J. Mater. Sci. Technol. 34 (2018) 1791-1798. doi:10.1016/J.JMST.2018.02.003.

[3] A.F. Guillermet, Assessing the thermodynamics of the FeCONi system using a calphad predictive technique, Calphad. 13 (1989) 1-22. doi:10.1016/0364-5916(89)90034-5.

[4] V. Raghavan, Co-Fe-Ni (cobalt-iron-nickel), J. Phase Equilibria. 15 (1994) 526-527. doi:10.1007/BF02649406.

[5] W. Koster, W.D. Haeha, The Real Constitutional Diagram and Equilibrium Adjustment in the Fe-Co-Ni Ternary System, Arch. Eisenhuttenwes. 40 (1969) 569-574.

[6] S. Widge, J.I. Goldstein, L. Uni-, Redetermination of the Fe-Rich Portion of the Fe-Ni-Co Phase Diagram, 8 (1977) 3-9.

[7] H. Xing, B. Zhao, Y. Wang, X. Zhang, Y. Ren, N. Yan, T. Gao, J. Li, L. Zhang, H. Wang, Rapid Construction of Fe-Co-Ni Composition-Phase Map by Combinatorial Materials Chip Approach, ACS Comb. Sci. 20 (2018) 127-131. doi:10.1021/acscombsci.7b00171.

[8] I.B. Borovskiy, I.D. Marchukova, Y.E. Ugaste, Local x-Ray spectranalysis of mutual diffusion in binary systems forming a continuous series of solid solutions, II. the systems Fe-Ni, Ni-Co, Ni-Pt and Co-Pt, 1967.

[9] Y.W. Cui, M. Jiang, I. Ohnuma, K. Oikawa, R. Kainuma, K. Ishida, Computational study of atomic mobility in Co-Fe-Ni ternary fcc alloys, J. Phase Equilibria Diffus. 29 (2008) 312-321. doi:10.1007/s11669-008-93419.

[10] B. Million, J. Růžičková, J. Velíšek, J. Vřeštál, Diffusion processes in the FeNi system, Mater. Sci. Eng. 50 (1981) 43-52. doi:10.1016/0025-5416(81)90084-7.

[11] Y.E. Ugaste, A.A. Kodentsov, F.J.J. Van Loo, Investigation of the interdiffusion and Kirkendall effect in the Co-Ni-Fe system: I. Redistribution of component concentrations in the diffusion zone and shift of inert markers, 2004.

[12] Y.E. Ugaste, A.A. Kodentsov, F.J.J. Van Loo, Compositional dependence of diffusion coefficients in the Co$\mathrm{Ni}, \mathrm{Fe}-\mathrm{Ni}$ and Co-Fe systems, 1999. 
[13] Y.E. Ugaste, A.A. Kodentsov, J. Priimets, Investigation of interdiffusion and kirkendall effect in the Co-NiFe system: III. Dependence of diffusion parameters on the initial conditions, Phys. Met. Metallogr. 110 (2010) 485-491. doi:10.1134/S0031918X10110086.

[14] T. Ustad, H. Sorum, Interdiffusion in the Fe-Ni, Ni-Co, and Fe-Co Systems, Phys. Status Solidi. 285 (1973) 285-293. doi:10.1017/CBO9781107415324.004.

[15] V. Ganesan, V. Seetharaman, V.S. Raghunathan, Interdiffusion in the nickel-iron system, Mater. Lett. 2 (1984) 257-262. doi:10.1016/0167-577X(84)90125-3.

[16] J.I. Goldstein, R.E. Hanneman, R.E. Ogilvie, Diffusion in the Fe-Ni system at 1 atm and 40 kbar pressure, 1965.

[17] T. Heumann, A. Kottmann, Über den Ablauf der Diffusionsvorgänge in Substitutionsmischkristallen, 1953.

[18] K.I. Hirano, R.P. Agarwala, B.L. Averbach, M. Cohen, Diffusion in cobalt-nickel alloys, J. Appl. Phys. 33 (1962) 3049-3054. doi:10.1063/1.1728564.

[19] K. Hirano, M. Cohen, Diffusion of Cobalt in Iron-Cobalt alloys, Mater. Trans. JIM. 13 (1972) 96-102.

[20] J. Kucera, K. Stransky, Diffusion in iron, iron solid solutions and steels, Mater. Sci. Eng. 52 (1982) 1-38. doi:10.1016/0025-5416(82)90067-2.

[21] B. Million, Diffusion von Kobalt in Ni-Co-Legierungen bei Temperaturen bis $1000^{\circ} \mathrm{C}$, Zeitschrift Für Met. 63 (1972) 484-489.

[22] B. Million, K. Ciha, J. Krejci, K. Stransky, Self-diffusion of Cobalt in coarse grained polycrystalline Ni-Co alloys at low temperature, Czechoslov. J. Physics, B. 22 (1972) 534-536. doi:10.1017/CBO9781107415324.004.

[23] B. Million, J. Kucera, J. Kučera, Concentration dependence of nickel diffusion in nickel-cobalt alloys, Czechoslov. J. Phys. 21 (1971) 161-171. doi:10.1007/BF01702804.

[24] Ü. Ugaste, J. Priimets, T. Laas, Dependence of Diffusion Paths on Thermodynamic Factors in Ternary Systems, Defect Diffus. Forum. 277 (2008) 119-124. doi:10.4028/www.scientific.net/DDF.277.119.

[25] Y.W. Cui, M. Jiang, I. Ohnuma, K. Oikawa, R. Kainuma, K. Ishida, Computational study of atomic mobility for fcc phase of Co-Fe and Co-Ni binaries, J. Phase Equilibria Diffus. 29 (2008) 2-10. doi:10.1007/s11669007-9238-z.

[26] J. Agren, Binary and multicomponent diffusion, in: Charact. Mater., 2012: pp. 1-16.

[27] Y. Yuan, L. Yang, D. Li, A. Tang, F.S. Pan, H. Seifert, N. Moelans, Diffusion Multiple Study of Co-Ni-Ti system at 1073K, Calphad Comput. Coupling Phase Diagrams Thermochem. (2018) Accepted.

[28] J.-C. Zhao, X. Zheng, D.G. Cahill, High-throughput diffusion multiples, Mater. Today. 8 (2005) 28-37. doi:10.1016/S1369-7021(05)71122-6.

[29] R.T.T. DeHoff, N. Kulkarni, The Trouble with Diffusion, Mater. Res. 5 (2002) 209-229. doi:10.1590/S151614392002000300002 
[30] Y. Yuan, D. Li, N. Moelans, Comments on "A numerical method to determine interdiffusion coefficients of Cu6Sn5 and Cu3Sn intermetallic compounds," Intermetallics. 69 (2016) 2015-2017. doi:10.1016/j.intermet.2015.10.015.

[31] Y. Yuan, D. Li, Y. Guan, H.J. Seifert, N. Moelans, Investigation of the diffusion behavior in Sn-xAg-yCu/Cu solid state diffusion couples, J. Alloys Compd. 686 (2016) 794-802. doi:10.1016/j.jallcom.2016.06.228.

[32] Y. Yuan, Y. Guan, D. Li, N. Moelans, Investigation of the diffusion behavior in Cu-Sn solid state diffusion couples, J. Alloys Compd. 661 (2016) 282-293. doi:10.1016/j.jallcom.2016.06.228.

[33] F.J.J.J. van Loo, Multiphase diffusion in binary and ternary solid-state systems, Prog. Solid State Chem. 20 (1990) 47-99. doi:10.1016/0079-6786(90)90007-3.

[34] C. Matano, On the relation between the diffusion-coefficients and concentrations of solid metals (The NickelCopper System), Japanese J. Phys. Trans. 16 (1933) 109-113.

[35] V.F. Von F. Sauer, Diffusion in binären Gemischen mit Volumenänderung, Zeitschrift Für Elektrochemie. 66 (1962) 353-363.

[36] Y. Iijima, K. Hirano, Interdiffusion in Cobalt-Nickel alloys, Japan Inst. Met. Mater. 35 (1971) 511-517.

[37] M. Badia, A. Vignes, Influence of structure changes produced by interdiffusion on interdiffusion coefficient and Kirkendall effect, Rev. Met. 66 (1969) 915-927. 